\title{
The Moderating Effect of Health on the Relationship between Involvement in Housework of Parents' and Parents-in-Law's Families and Happiness of Middle-Aged Women in Taiwan
}

\author{
Ching-Fen Lee1, Shain-May Tang2* \\ ${ }^{1}$ Department of Early Childhood Educare, Ching Kuo Institute of Management \& Health, Taiwan \\ ${ }^{2}$ Department of Living Science, National Open University, Taiwan \\ Email: 062888@yahoo.com.tw, *smtang@mail.nou.edu.tw
}

How to cite this paper: Lee, C.-F. and Tang, S.-M. (2020) The Moderating Effect of Health on the Relationship between Involvement in Housework of Parents' and Parents-in-Law's Families and Happiness of Middle-Aged Women in Taiwan. Health, 12, 1217-1240.

https://doi.org/10.4236/health.2020.129089

Received: August 28, 2020

Accepted: September 22, 2020

Published: September 25, 2020

Copyright $\odot 2020$ by author(s) and Scientific Research Publishing Inc. This work is licensed under the Creative Commons Attribution International License (CC BY 4.0).

http://creativecommons.org/licenses/by/4.0/ Open Access

\begin{abstract}
Background: This study aims to investigate the moderating effect of family health status on the relationship between involvement in housework of two-side parents' families and happiness of middle-aged women in Taiwan. Methods: The data used in this study were gathered from the Ministry of Science and Technology's program-“Intergenerational expectation and collaboration: an inquiry on a new mechanism of continued tradition across generations". The subjects $(n=512)$ were restricted to aged $45-64$ years old who live with spouses and have children, and one of whose parent or parent-in-law is still alive. Multiple regression analysis was used to investigate how family health status moderates the relationship between involvement in housework of parents' families and parents-in-law's families and the happiness of middle-aged women. Results: 1) Family health status is positively correlated with their happiness. 2) The parents' health status can moderate the relationship between involvement in housework of parents' families and happiness of the middle-aged women. 3) The middle-aged women's health status can moderate the relationship between involvement in housework of parents-in-law's families and those women's happiness, and fathers-in-law's health status can moderate the relationship between husbands' involvement in housework of the women's parents-in-law's families and women's happiness. Conclusions: In future, when policies and programs related to the well-being of middle-aged women are being planned, the effect of women's health and family health status shall be considered as a key to improve those
\end{abstract}


women's quality of life.

\section{Keywords}

Middle-Aged Women, Intergenerational Support, Housework, Family Health, Happiness

\section{Introduction}

Happiness is a kind of evaluation of subjective feelings on life which are held by individuals [1], and is also one of the core concepts affecting human health and quality of life [2] as well as constructing positive psychology. Therefore, the discussion on topics concerning happiness has attracted people's attention with the rise of positive psychology [3]. However, the question must be asked, what are the factors that affect happiness? Previous studies have found that personal characteristics (such as income, education level, etc.) [4], family relationships [5], adaptation to life [6], self-assessment of health [7], etc. can affect individuals' sense of happiness. Will gender differences in individuals' psychological well-being be derived with age? It has been found that the unhappiness index of women increases with their age [8]; this may be because women live longer than men, and women, one the other hand, are more likely to rely on others' assistance in housework and health care due to degradation of their physiological function at the later stage of their life, at which point their happiness level declines accordingly [9]. The aforementioned findings are related to exploring the happiness of the elderly under care, but what are the factors correlating with the happiness in life of the middle-aged women who are the main caregivers of the family? What role does family health status play in terms of this issue? More specifically, the background of this study is that the baby boomers who have promoted social and economic development have gradually become middle-aged or old, and these new generation women hold contrasting values and have different social and economic statuses from those of the traditional old people [10]. Are the happiness feelings of such women still affected by the female characters in the context of traditional culture? Or are there other factors that affect their happiness? The above questions constitute what this paper intends to discuss.

In addition, the issues in regard to the division of housework are explored mainly based on the traditional theoretical frameworks of housework division, such as the time availability perspective, the gender role attitude, the resources perspective, and other theoretical construction perspectives on the division of labor mainly based on husband and wife in the western core families [11]. Due to the close relationship between generations in Chinese families, housework support across generations is quite common [10]; therefore, the traditional western theory of housework division is not entirely applicable to Chinese families in the context of familial culture. Since women are mainly responsible for 
housework at home, it is not difficult to imagine that in the case of "burning the candle at both ends"; the frequency and status of housework performance is usually negatively correlated with their happiness [12]; at this time, if spouses provide more housework support, it can help women relieve their housework pressure and improve their happy feelings [12]. Therefore, the participation of men in housework should also be included in the discussion. Diener et al. [13] pointed out that cultural context is the key factor in guiding individuals to construct their life needs and cognition and then affect their happiness. Thus, when individuals' happiness is examined, discussion on how people living in the cultural context pile up their own emotions cannot be ignored. The previous studies have pointed out that the most commonly discussed issue on intergenerational relations in Taiwan is the functional solidarity perspective that emphasizes the exchange or assistance of family members through resources such as money, housework and emotions [14]. Besides this, some studies have put forth the belief that children's support and care for their parents in life is not only the manifestation of intergenerational functional solidarity, but also the internalization and awareness of embedding filial piety in, and regarding it as, the norm of family solidarity [15]; that is, the intergenerational interaction model framed by filial piety is a cultural value indicator that cannot be ignored, which not only influences the thoughts of children, but also influences children's behaviors.

Besides, both the intergenerational solidarity theory mentioned by Bengtson and Schrader [16] in the past and the perspectives often mentioned recently, such as mutual filial piety in which gratitude between generations is emphasized by the dual filial piety model and the authoritative filial piety in accordance with role and class norms, are closely linked with gender [17]. The reason is that the intergenerational family interaction in Chinese culture highlights the role differences of people with different genders in families and society due to the gender division of labor. In other words, in traditional values, women are assigned to the family and thus are given the role of family caregivers, responsible for maintaining family relations, performing intergenerational housework, and taking care of household affairs [18]. However, when only women in the family undertake all the important and trivial affairs, it cannot be determined whether these are the sweet burdens or the responsibilities for the family in despair for women. This is also the reason why the present study focuses on women when analyzing the impact of housework support on happiness across generations.

Different from previous studies focusing on the relationship between married women and their parents-in-law's families [19], this study holds the belief that the most significant change for women in Taiwan is that they are not limited to the traditional values of "daughters are water poured out of the family after they get married" [20]; that is, the relationship between women and their parents' families does not end due to marriage, and on the other hand they become more grateful and are sincere and willing to provide assistance to parents' families 
[21]. In contrast, although there is no upbringing relationship between women and their parents-in-law's families, there is an expectation from the cultural norms that the married daughters-in-law should show filial obedience to, and take care of, their husbands' family [15]. When middle-aged women are faced with the relationship between intergenerational housework support and their happiness, on the one hand they are grateful for their parents' families due to consanguinity, and on the other hand they have to comply with the culture context in relation to parents-in-law's families based on female rules. In this case, what may happen? The present study analyzes the sample in the aspect of parents' families and that of parents-in-law's families respectively to understand the differences.

It can be seen from the above that the care for the elderly parents is made the responsibility of the family. However, in the past studies, it has also been mentioned that due to the influence of the norms of the father-son axis, the elderly parents tend to rely on their sons for life care and support in old age, but in fact it is the women in the family, namely the daughters-in-law, who are in charge of such responsibilities. In addition, another question must also be asked, namely has the phenomenon that women should take the responsibility of the family but do not have the right to the family [22] caused a qualitative change in the distribution of rights and responsibilities between husband and wife in intergenerational care as the optimistic and positive baby boomers whose family care views are different from the traditional become the masters of their own affairs [23]? Is the attitude of husbands toward intergenerational support different from the traditional hands-off approach? And does their intergenerational support provision in real life affect the happiness of middle-aged women? Based on such questions, this study also discusses the relationship between intergenerational housework support from husbands and middle-aged women's happiness, so as to have a more complete analysis and understanding of the factors affecting middle-aged women's happiness.

In addition, physical health status for the middle-aged and the elderly is a symbol of their ability to take care of themselves in daily life and participate in society [24]. Especially for some middle-aged and old people, physical health represents whether they can provide assistance to the family or its members, and also represents the stability of their status in the family [24]. Moreover, it has been found in previous studies that there is a close relationship between individual health and housework participation and happiness [1]. Lee and Tang [25] also found that the relationship between housework and happiness of middle-aged and elderly women can be affected by the health of their spouses. Therefore, when it comes to the interaction between the relationship of housework with happiness and other variables, it is insufficient to understand it only from the relationship perspective [26]. That is to say, for the middle-aged and elderly families that gradually enter the contradiction period, the fact that their children start to leave home may mean a little relaxation of the housework for 
the husband and wife [27]. However, with the improvement of environmental health conditions and the increase in average life expectancy of humans, the time span of interaction and care between middle-aged and elderly couples, as well as the life support and care period for their elderly parents, are also extended [28]. The previous studies found that adult children often suffer from pressure due to lack of others' assistance when they take care of their parents in life, resulting in a decline in their psychological well-being [29]. Accordingly, when the factors that affect the happiness of middle-aged women are discussed, the health status of family members (such as spouses, parents and parents-in-law) should be included. Therefore, in addition to the influence of intergenerational housework sharing on happiness of middle-aged women under the context of Chinese culture and the health status of themselves and their husbands, this study also includes the health status of parents and parents-in-law in discussion, and compares the differences between the roles of daughters-in-law and daughters.

In view of forgoing discussions, this study proposes four main hypotheses:

Hypothesis 1: when the personal background variables are controlled, middle-aged women's provision of intergenerational housework support may significantly affect their happiness.

Hypothesis 2: when the personal background variables are controlled, husbands' provision of intergenerational housework support may significantly affect middle-aged women's happiness.

Hypothesis 3: when the personal background variables are controlled, the moderating effect of family health status on the relationship between middle-aged women's provision of intergenerational housework support and their happiness may be different due to the differences in parents' families and parents-in-law's families.

Hypothesis 4: when the personal background variables are controlled, the moderating effect of family health status on the relationship between husbands' provision of intergenerational housework support and middle-aged women's happiness may be different due to the differences in parents' families and parents-in-law's families.

In addition, in order to avoid the interference of other factors affecting the happiness feelings of middle-aged women, variables such as age, work status, gender role attitude, and social and economic status, are regarded as control variables in the research model in this study. In terms of age, although previous studies hold different views on the relationship between age and individual psychological well-being, it is undeniable that age is one of the factors affecting the psychological well-being of middle-aged and elderly people [30]. Moreover, since work status is a reflection of individual economic conditions, when the jobs of the middle-aged and old people terminate because of retirement, such termination of jobs means a shortage in the economy [31] and a lack of free choice and sense of security at the material level in life, resulting in their psychological well-being be- 
ing relatively lower than that of the working people [32]. Gender role is the basis and expectation of individuals' behaviors and role-playing in society endowed by culture, and thus it is not difficult to understand that individual psychological well-being may also be affected by gender role attitude [33]. Finally, Botha et al. [4] found that socioeconomic status is positively correlated with individual happiness, that is, people with higher socioeconomic status have a higher happiness index.

\section{Methods}

\subsection{Design}

The purpose of this study is to analyze the effect of intergenerational housework support on happiness of middle-aged women and the moderating effect of family health status. Part of the research topics of the Ministry of Science and Technology's program- "family history in aged society in Taiwan: discussion of positive transformation-intergenerational expectation and collaboration: an inquiry on a new mechanism of continued tradition across generations", is adopted as the data source, and the program has passed the review of the human subject research ethics committee of Fu Jen Catholic University before the implementation of the program (IRB approval number: C104027). As for the selection of research samples, used purposive sampling, the subjects are married women living in Taiwan who have children and one of whose parents or parents-in-law is still alive, while their age range is $45-64$. After the original data in the questionnaires were encoded, logged and documented, SPSS 18 for Windows statistical software was used to analyze the data, and based on the research results, the research conclusions and suggestions were put forward.

\subsection{Participants}

The data used in this study were collected in schools, government agencies, private companies, neighborhoods, private parties and other places by 36 contacts. For sample heterogeneity, it was stipulated that no more than 30 questionnaires should be distributed and collected by each contact. It was also deemed that all questionnaires should be handled anonymously and an envelope should be attached to state explanations and to remind the respondents of matters needing attention. In this study, a total of 540 questionnaires were obtained, and among them 512 questionnaires were valid, excluding those not meeting the requirements and those in which the questions were not answered or the basic data were missed.

A total of 512 women are included in the sample for analysis in this study. The average age of the respondents is 51.54 years old. In terms of work status, the women with full-time jobs account for $65.8 \%(\mathrm{n}=337)$ and are in the majority, while those without jobs account for $17.4 \%(n=89)$, and those with part-time jobs constitute $16.8 \%(n=86)$. As for gender role attitude, $47 \%(n=240)$ of the women generally agree with the view of "husbands should earn enough money 
to support the family", and are in the majority, while $30.1 \%(\mathrm{n}=154)$ of them generally disagree with the view, $18.2 \%(n=93)$ strongly agree with it, and $4.7 \%$ $(\mathrm{n}=24)$ strongly disagree with it. In terms of social and economic status, $65.2 \%$ ( $n=334)$ of them are similar to other families in the society and are in the majority, $21.5 \%(n=110)$ of them have a higher status than other families, $10.0 \%(n$ $=51)$ have a lower status than other families, $2.1 \%(n=11)$ have a much higher status than other families, and $1.2 \%(n=6)$ have a much lower status than other families.

In terms of assistance provision in housework of the middle-aged women to their parents' families, $75.2 \%(n=385)$ of them do not provide housework support to their parents' families and are in the majority, while $24.8 \%(n=127)$ of them do provide assistance. As for assistance provision in housework of the middle-aged women's husbands to the women's parents' families, 97.3\% ( $\mathrm{n}=$ 498) of the husbands do not provide housework support to the middle-aged women's parents' families and are in the majority, while $2.7 \%(n=14)$ of them do provide assistance. Regarding the aspect of assistance provision in housework of the middle-aged women to their parents-in-law's families, 69.7\% ( $n=357$ ) of them do not provide housework support to their parents-in-law's families and are in the majority, while $30.3 \%(n=155)$ do provide housework support. As for assistance provision in housework of the middle-aged women's husbands to the women's parents-in-law's families, $80.9 \%(n=414)$ of the husbands do not provide housework support to the middle-aged women's parents-in-law's families, and $19.1 \%(n=98)$ of them do provide housework support.

In regard to family health status, $40.4 \%(n=205)$ of the middle-aged women's husbands are in a normal health condition and are in the majority, $39.4 \%$ ( $\mathrm{n}=$ $200)$ of the husbands are in good health, $15.4 \%(n=78)$ of them are in an excellent health condition, $4.3 \%(n=22)$ are in a poor condition, and $0.6 \%(n=3)$ are in extremely poor health. Regarding the middle-aged women's health condition, $41.3 \%(n=211)$ of the middle-aged women are in a normal health condition and are in the majority, $38.4 \%(n=196)$ of them are in good health, $14.5 \%(n=74)$ are in excellent condition, $5.5 \%(n=28)$ are in poor condition, and $0.4 \%(n=2)$ are in extremely poor health. Regarding the health condition of the middle-aged women's fathers, $46.2 \%(n=114)$ of the fathers are in a normal health condition and are in the majority, while $23.5 \%(\mathrm{n}=58)$ are in a poor condition, $19.0 \%(\mathrm{n}=$ $47)$ of them are in good health, $6.9 \%(n=17)$ are in extremely poor health, and $4.5 \%(n=11)$ are in an excellent health condition. In terms of the health condition of the middle-aged women's mothers, $39.7 \%(n=163)$ of the mothers are in a normal health condition and are in the majority, while $25.3 \%$ ( $n=104)$ of them are in poor condition, $21.7 \%(\mathrm{n}=89)$ of them are in good health, $8.0 \%(\mathrm{n}$ $=33)$ are in excellent health condition, and $5.4 \%(n=22)$ are in extremely poor health. As for the health condition of the middle-aged women's fathers-in-law, $39.1 \%(n=90)$ of the fathers-in-law are in a normal health condition and are in the majority, $30.0 \%(n=69)$ are in poor condition, $16.1 \%(n=37)$ of them are in 
good health, $7.8 \%(\mathrm{n}=18)$ are in excellent condition, and $7.0 \%(\mathrm{n}=16)$ are in extremely poor health. In terms of the health condition of the middle-aged women's mothers-in-law, $43.7 \%(n=156)$ of the mothers-in-law are in a normal health condition and are in the majority, $20.7 \%(\mathrm{n}=74)$ are in poor condition, $19.6 \%(n=70)$ of them are in good health, $8.7 \%(n=31)$ are in extremely poor health, and 7.3\% $(\mathrm{n}=26)$ are in excellent condition (Table 1$)$.

\subsection{Measures}

\subsubsection{Independent Variable: Intergenerational Housework Support}

The intergenerational housework support in this study includes four items: the housework support of the middle-aged women to their parents' families and their parents-in-law's families, and the housework support of these women's husbands to the women's parents' families and parents-in-law's families. The items are described separately as follows: in terms of housework support provision of the middle-aged women for their parents' families or parents-in-law's families, the question is set as "who is responsible for the housework of parents' families mainly?" or "who is responsible for the housework of parents-in-law's families mainly?"; indeed, in the questionnaire these questions are adopted for measurement. When the option selected by a middle-aged woman is that she does the housework of her parents' families or her parents-in-law's families, the score is " 1 "; when the choice is that she does not do the housework of her parents' families or her parents-in-law's families, the score is " 0 ". In terms of housework support provision of the middle-aged women's husbands for women's parents' families or parents-in-law's families, the question is set as "who does the housework of parents' families mainly?" or "who does the housework of parents-in-law's families mainly?"; in the questionnaire, these are also adopted for measurement. When the choice is that the s or parents-in-law's families, the score is " 1 "; when the choice is that the spouse does not do the housework of parents' families or parents-in-law's families, the score is " 0 ".

\subsubsection{Dependent Variable: Happiness}

Amato and Booth [34] pointed out that the most direct and effective way to measure happiness is to directly ask the respondents the question "do you feel happy?", so as to assess individuals' subjective feelings about life as a whole. Based on this viewpoint, the present study uses a single question- "do you feel happy about your current life as a whole?"-to measure the happiness degree of the respondents. The score ranges from " 1 " (very unhappy) to " 5 " (very happy). A higher score means that the respondents have a higher happiness level in their current life.

\subsubsection{Moderator Variable: Family Health Status}

The moderator variable of this study is family health status, including the health status of middle-aged women themselves, their spouses, parents and parents-in-law. Their health status is measured by the items of "health status of yourself/your 
Table 1. Sample characteristics.

\begin{tabular}{|c|c|c|}
\hline & n (\%) & $\mathrm{M}(\mathrm{SD})$ \\
\hline Age & & $51.54(5.03)$ \\
\hline \multicolumn{3}{|l|}{ Work status } \\
\hline Full-time & $337(65.8 \%)$ & \\
\hline Part-time & $86(16.8 \%)$ & \\
\hline Unemployed & $89(17.4 \%)$ & \\
\hline Gender role attitude & & $2.79(0.79)$ \\
\hline Strongly disagree & $24(4.7 \%)$ & \\
\hline Disagree & $154(30.1 \%)$ & \\
\hline Agree & $240(47.0 \%)$ & \\
\hline Strongly agree & $93(18.2 \%)$ & \\
\hline SES (compared with other families in the society) & & $3.13(0.66)$ \\
\hline Very much lower than most households & $6(1.2 \%)$ & \\
\hline Lower than most households & $51(10.0 \%)$ & \\
\hline About the average & $334(65.2 \%)$ & \\
\hline Higher than most households & $110(21.5 \%)$ & \\
\hline Very much higher than most households & $11(2.1 \%)$ & \\
\hline \multicolumn{3}{|l|}{ Respondent's support to her parent's housework } \\
\hline No & $385(75.2 \%)$ & \\
\hline Yes & $127(24.8 \%)$ & \\
\hline \multicolumn{3}{|l|}{ Spouse's support to his parent in-law's housework } \\
\hline No & $498(97.3 \%)$ & \\
\hline Yes & $14(2.7 \%)$ & \\
\hline \multicolumn{3}{|l|}{ Respondent's support to her parent in-law's housework } \\
\hline No & $357(69.7 \%)$ & \\
\hline Yes & $155(30.3 \%)$ & \\
\hline \multicolumn{3}{|l|}{ Spouse's support to his parent's housework } \\
\hline No & $414(80.9 \%)$ & \\
\hline Yes & $98(19.1 \%)$ & \\
\hline Assessment of spouse's health status & & $3.65(0.81)$ \\
\hline Very poor & $3(0.6 \%)$ & \\
\hline Poor & $22(4.3 \%)$ & \\
\hline Neither good nor poor & $205(40.4 \%)$ & \\
\hline Good & $200(39.4 \%)$ & \\
\hline Very good & $78(15.4 \%)$ & \\
\hline Assessment of self-health status & & $3.61(0.81)$ \\
\hline Very poor & $2(0.4 \%)$ & \\
\hline
\end{tabular}




\section{Continued}

\begin{tabular}{|c|c|c|}
\hline Poor & $28(5.5 \%)$ & \\
\hline Neither good nor poor & $211(41.3 \%)$ & \\
\hline Good & $196(38.4 \%)$ & \\
\hline Very good & $74(14.5 \%)$ & \\
\hline Assessment of father's health status & & $2.91(0.94)$ \\
\hline Very poor & $17(6.9 \%)$ & \\
\hline Poor & $58(23.5 \%)$ & \\
\hline Neither good nor poor & $114(46.2 \%)$ & \\
\hline Good & $47(19.0 \%)$ & \\
\hline Very good & $11(4.5 \%)$ & \\
\hline Assessment of mother's health status & & $3.02(1.00)$ \\
\hline Very poor & $22(5.4 \%)$ & \\
\hline Poor & $104(25.3 \%)$ & \\
\hline Neither good nor poor & $163(39.7 \%)$ & \\
\hline Good & $89(21.7 \%)$ & \\
\hline Very good & $33(8.0 \%)$ & \\
\hline Assessment of father in-law's health status & & $2.88(1.02)$ \\
\hline Very poor & $16(7.0 \%)$ & \\
\hline Poor & $69(30.0 \%)$ & \\
\hline Neither good nor poor & $90(39.1 \%)$ & \\
\hline Good & $37(16.1 \%)$ & \\
\hline Very good & $18(7.8 \%)$ & \\
\hline Assessment of mother in-law's health status & & $2.96(1.02)$ \\
\hline Very poor & $31(8.7 \%)$ & \\
\hline Poor & $74(20.7 \%)$ & \\
\hline Neither good nor poor & $156(43.7 \%)$ & \\
\hline Good & $70(19.6 \%)$ & \\
\hline Very good & $26(7.3 \%)$ & \\
\hline
\end{tabular}

spouse/your mother/your father/your spouse's mother/your spouse's father". The score ranges from " 1 " (extremely poor) to " 5 " (excellent). A higher score means a better health condition.

\subsubsection{Control Variable}

In order to avoid the influence of other variables, age, work status, gender role attitude, social and economic status are considered as control variables. The age is determined based on the question "how old are you? years old".

The work status of the respondents is determined by the question "what is your current work status?”. There are three options: full-time job, part-time job 
and no job.

The gender role attitude is measured by the statement "husbands should make enough money to support their family" in the questionnaire. The score ranges from " 1 " (strongly disagree) to " 5 " (strongly agree). A higher score represents a more traditional attitude toward gender roles.

The social and economic status is measured by using, within the questionnaire, the following interrogative: "compared with other families in the society, is your family income higher, lower, or similar?". The score ranges from " 1 " (much lower) to " 5 " (much higher). A higher score means they feel that they have a higher social economic status.

\subsection{Statistical Analysis}

The SPSS 18 statistical software package is used for data statistics and analysis in this study. In terms of data analysis, the main statistical methods adopted are as follows:

1) Descriptive statistics: with regard to the description of basic data, the research results are presented by frequency distribution, average and standard deviation.

2) Multiple linear regression analysis: this is adopted to explore the moderating effect of family health status on the relationship between intergenerational housework support and happiness of middle-aged women. Before the test for moderator variables, in order to avoid the so-called "multi-collinearity" problem, which may arise because the interaction variable is obtained by multiplication of the independent variable and the moderator variable, the average of the continuous variable to be moderated is first subtracted from it, whether the continuous variable is an independent variable or a moderator variable, and then the mean-centering is obtained by multiplying the interaction items to solve the multi-collinearity problem [35].

\section{Results}

\subsection{The Relationship between Intergenerational Housework Support as Well as Family Health Status and Happiness of Middle-Aged Women}

\subsubsection{The Relationship between Intergenerational Housework Support as Well as Family Health Status of Parents' Families and Happiness of Middle-Aged Women}

In this study, regression analysis is used to investigate the relationship between variables related to parents' families in the sample and happiness of the middle-aged women. The results show that the $\mathrm{F}$ value of the whole model is 4.18 , reaching the significance level 0.001 , which indicates that this model has explanatory power. In terms of the variables of intergenerational housework support, there is no correlation between the middle-aged women's involvement in housework for their parents' families $(\beta=0.01, \mathrm{p}>0.05)$, as well as their husbands' involvement in housework for their parents' families $(\beta=-0.11, \mathrm{p}>0.05)$, and 
happiness of middle-aged women. However, as for family health, there is a significant correlation between their own health $(\beta=0.26, \mathrm{p}<0.01)$, as well as their mothers' health $(\beta=0.16, \mathrm{p}<0.05)$, and the middle-aged women's happiness; that is, when the respondents rate themselves or their mothers as healthier, the happiness degree of them is higher (Table 2).

\subsubsection{The Relationship between Intergenerational Housework Support, as Well as Family Health Status of Parents-in-Law's Families, and Happiness of Middle-Aged Women}

For the parents-in-law's families, the results show that the $\mathrm{F}$ value of the whole model is 3.92, reaching the significance level 0.001, which indicates that the model has explanatory power. In terms of the variables, there is no correlation between the middle-aged women's involvement in housework for their parents-in-law's

Table 2. Regression analysis for middle-aged women perceived happiness (Original family).

\begin{tabular}{|c|c|c|c|c|c|}
\hline Variable & B & Beta & $\mathrm{t}$ & p-value & VIF \\
\hline \multicolumn{6}{|l|}{ Control variables } \\
\hline Age & 0.03 & 0.15 & $2.41^{\star}$ & 0.017 & 1.10 \\
\hline \multicolumn{6}{|l|}{ Work status $(0=$ Full-time $)$} \\
\hline Part-time & 0.05 & 0.02 & 0.37 & 0.712 & 1.13 \\
\hline Unemployed & -0.07 & -0.03 & -0.46 & 0.649 & 1.22 \\
\hline Gender role attitude & -0.05 & -0.05 & -0.74 & 0.459 & 1.10 \\
\hline SES (compared with other families in the society) & 0.23 & 0.18 & $2.88^{\star *}$ & 0.004 & 1.07 \\
\hline \multicolumn{6}{|l|}{ Independent variable } \\
\hline Respondent's support to her parent's housework & 0.01 & 0.01 & 0.07 & 0.941 & 1.19 \\
\hline Spouse's support to his parent in-law's housework & -0.47 & -0.11 & -1.02 & 0.308 & 2.92 \\
\hline \multicolumn{6}{|l|}{ Moderator variable } \\
\hline Assessment of spouse's health status & 0.09 & 0.08 & 0.98 & 0.328 & 1.81 \\
\hline Assessment of self-health status & 0.27 & 0.26 & $3.12^{\star *}$ & 0.002 & 1.85 \\
\hline Assessment of father's health status & -0.03 & -0.03 & -0.39 & 0.697 & 1.52 \\
\hline Assessment of mother's health status & 0.14 & 0.16 & $2.12^{*}$ & 0.036 & 1.53 \\
\hline Respondent's support to her parent's housework $\times$ Assessment of self-health status & 0.13 & 0.05 & 0.65 & 0.517 & 1.76 \\
\hline Spouse's support to his parent in-law's housework $\times$ Assessment of spouse's health status & -1.42 & -0.19 & -1.14 & 0.255 & 7.47 \\
\hline Respondent's support to her parent's housework $\times$ Assessment of father's health status & -0.44 & -0.21 & $-2.48^{*}$ & 0.014 & 1.95 \\
\hline Spouse's support to his parent in-law's housework $\times$ Assessment of father's health status & 0.05 & 0.01 & 0.08 & 0.936 & 2.04 \\
\hline Respondent's support to her parent's housework $\times$ Assessment of mother's health status & 0.32 & 0.17 & $2.20^{*}$ & 0.029 & 1.64 \\
\hline Spouse's support to his parent in-law's housework $\times$ Assessment of mother's health status & 0.55 & 0.11 & 0.76 & 0.448 & 5.59 \\
\hline $\mathrm{R}^{2}$ & 0.27 & & & & \\
\hline Adjusted $\mathrm{R}^{2}$ & 0.20 & & & & \\
\hline F-value & $0.418^{\star * *}$ & & & 0.000 & \\
\hline
\end{tabular}

${ }^{*} \mathrm{p}<0.05,{ }^{* *} \mathrm{p}<0.01,{ }^{* *} \mathrm{p} \leq 0.001$. 
families $(\beta=0.09, \mathrm{p}>0.05)$, as well as their husbands' involvement in housework for their parents-in-law's families $(\beta=0.02, \mathrm{p}>0.05)$, and happiness of the middle-aged women. However, with regard to family health, there is a correlation between their own health $(\beta=0.24, \mathrm{p}<0.01)$, as well as their husbands' health $(\beta=0.24, \mathrm{p}<0.05)$, and the middle-aged women's happiness; that is, when the respondents rate themselves or their husbands as healthier, the happiness degree of them is higher (Table 3 ).

\subsection{The Moderating Effect of Family Health Status on the Relationship between Intergenerational Housework Support and Happiness of Middle-Aged Women}

\subsubsection{The Moderating Effect of Family Health Status on the Sample in the Aspect of Parents' Families}

With regard to the moderating effect of family health status on the sample in the aspect of parents' families, only Assessment of fathers' health $(\beta=-0.21, \mathrm{p}<$ $0.05)$ and Assessment of mothers' health $(\beta=0.17, \mathrm{p}<0.05)$ can respectively moderate the relationship between middle-aged women's involvement in housework for their parents' families and their happiness. However, Assessment of spouse's health status $(\beta=-0.19, \mathrm{p}>0.05)$ cannot moderate the relationship between husbands' involvement in the housework of the women's parents' families and middle-aged women's happiness; and neither can the middle-aged women's health status $(\beta=0.05, \mathrm{p}>0.05)$ moderate the relationship between their involvement in the housework of their parents' families and their happiness. Moreover, neither the health status of fathers $(\beta=0.01, \mathrm{p}>0.05)$ nor that of mothers $(\beta=0.11, \mathrm{p}>0.05)$ can moderate the relationship between husbands' involvement in the housework of the women's parents' families and middle-aged women's happiness (Table 2).

In order to understand more clearly the influence of assessment of fathers' health and assessment of mothers' health on the relationship between middle-aged women's involvement in the housework of their parents' families and their happiness, in this study, simple effect analysis is conducted respectively in relation to the aforementioned interaction variables which reach the significance level. Before the simple effect analysis, the health status of the fathers, the health status of the mothers, and their housework support to their parents' families are classified. The classification of the health status of family members is conducted by categorizing the options " 1 (extremely poor)" and " 2 (poor)" for the title "the health of yourself and your family" in the questionnaire as "unhealthy", categorizing option "3 (normal)" as "normal", and categorizing options "4 (good)" and "5 (excellent)" as "healthy". In regard to the middle-aged women's housework support to their parents' families, option " 0 (no)" for the question "who is responsible for the housework of parents' families mainly?" is classified as "the middle-aged women do not provide assistance in the housework of their parents' families", and option " 1 (yes)" is classified as "the middle-aged women do provide assistance in the housework of their parents' families". 
It can be seen from the results that when parents' health status is different, the impact will be different; when their mothers' health status is normal, the middle-aged women who help their parents complete housework also have a higher happiness degree. When their mothers' health status is unhealthy or healthy, the trend is that the respondents who help their parents' families complete housework have a lower happiness degree; and in cases where the health status of their mothers is unhealthy, the middle-aged women who help their mothers complete housework achieve the lowest happiness degree (Figure 1). Besides, when their fathers' health status is unhealthy, the middle-aged women who help their parents do housework also achieve a higher happiness degree. Moreover, in the case where their fathers' health status is normal or healthy, the respondents who do housework for their parents tend to have a lower happiness degree, and when their fathers' health status is healthy, the middle-aged women who do housework for their parents achieve the lowest happiness degree (Figure 2).

\subsubsection{The Moderating Effect of Family Health Status on the Sample in the Aspect of Parents-in-Law's Families}

With regard to the moderating effect of family health status on the sample in the aspect of parents-in-law's families, it can been seen from the results that the health status of the middle-aged women $(\beta=0.17, \mathrm{p}<0.05)$ can moderate the relationship between their involvement in the housework of their parents-in-law's families and their happiness; moreover, the health status of fathers-in-law of the middle-aged women $(\beta=-0.18, \mathrm{p}<0.05)$ can moderate the relationship between husbands' involvement in the housework of parents-in-law's families and the middle-aged women's happiness. However, mothers-in-law's health status ( $\beta=$ $-0.00,-0.04, \mathrm{p}>0.05$ in both cases) cannot moderate the relationship between

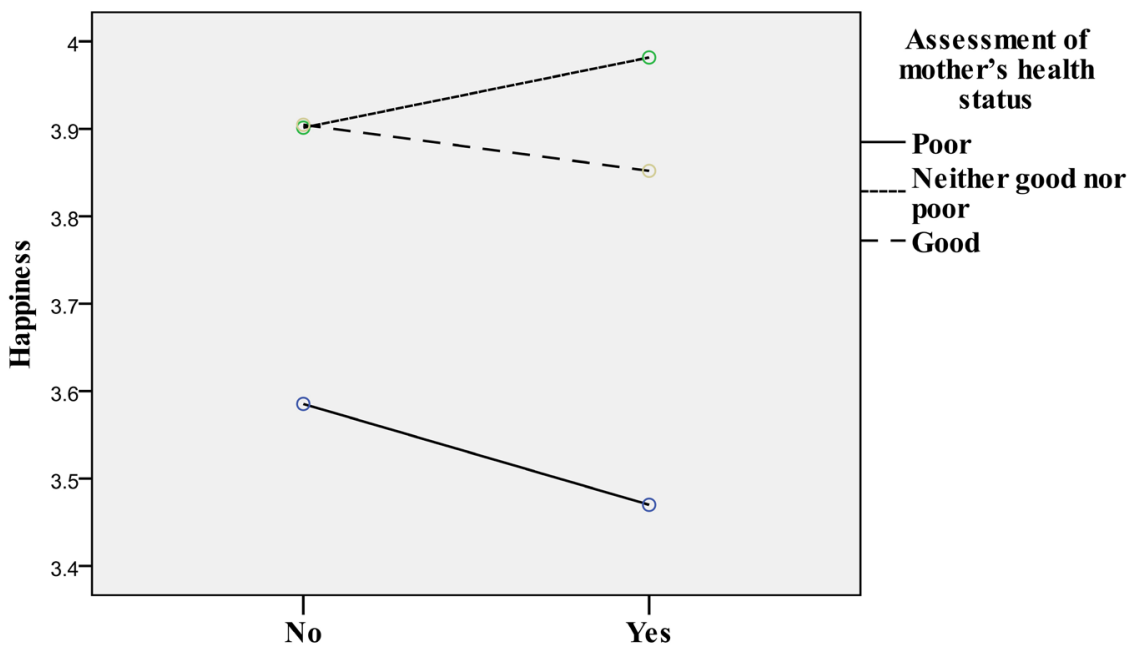

Respondent's support to her parent's housework

Mean of Covariates: Age $=49.80$, Work status $($ Part-time $)=0.18$, Work status(Unemployed) $=0.15$, Gender role attitude $=2.81$, SES (compared with other families in the society) $=3.09$, Assessment of self-health status $=3.61$, Assessment of spouse' $s$ health status $=3.65$, Assessment of father' $s$ health status $=2.93$, Spouse' $s$ support to his parent in-law' s housework $=0.04$

Figure 1. Interaction effect of respondent's support to her parent's housework and assessment of mother's health status. 


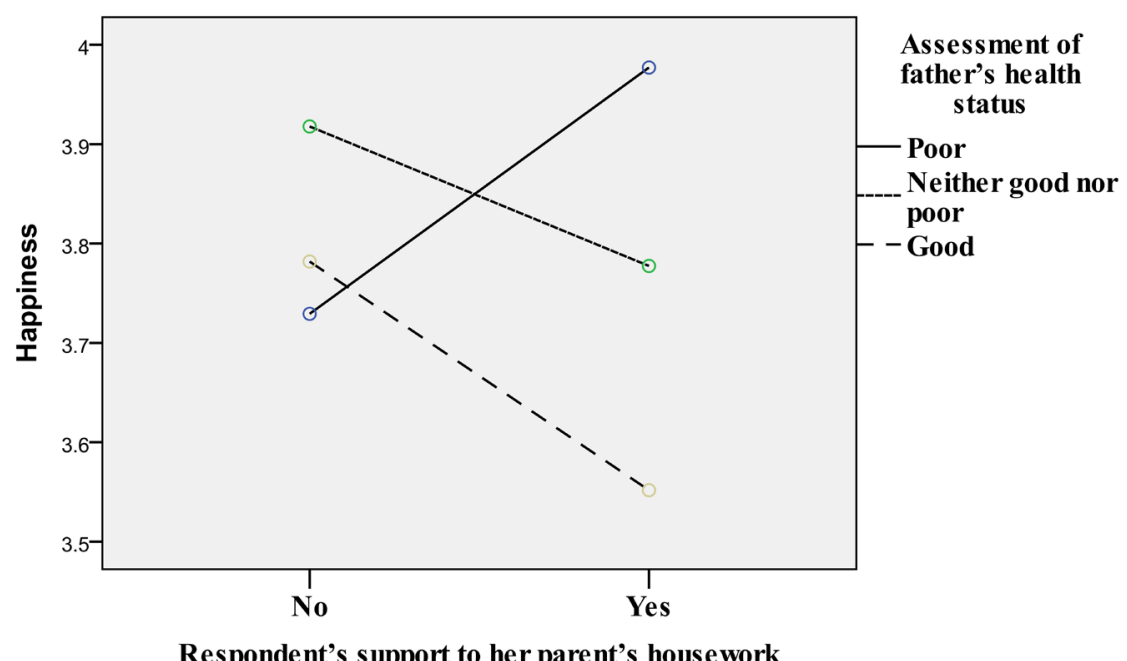

Respondent's support to her parent's housework

Mean of Covariates: Age $=49.80$, Work status $($ Part-time $)=0.18$, Work status(Unemployed) $=\mathbf{0 . 1 5}$, Gender role attitude $=\mathbf{2 . 8 1}$, SES $($ compared with other families in the society) $=3.09$, Assessment of self-health status $=3.61$, Assessment of spouse's health status $=3.65$, Assessment of mother's health status $=\mathbf{3 . 0 8}$, Spouse's support to his parent in-law's hous ework $=0.04$

Figure 2. Interaction effect of respondent's support to her parent's housework and assessment of father's health status.

intergenerational housework support (including the middle-aged women's involvement in the housework of parents-in-law's families, and husbands' involvement in the housework of parents-in-law's families) and middle-aged women's happiness; in addition, the health status of husbands of the middle-aged women $(\beta=0.08, \mathrm{p}>0.05)$ cannot moderate the relationship between husbands' involvement in the housework of parents-in-law's families and the middle-aged women's happiness; and the health status of the fathers-in-law of the middle-aged women $(\beta=-0.05, \mathrm{p}>0.05)$ cannot moderate the relationship between the middle-aged women's involvement in the housework of parents-in-law's families and the middle-aged women's happiness (Table 3).

In order to understand more clearly the respective effects of the middle-aged women's health and their fathers-in-law's health on the relationship between variables related to intergenerational housework support and happiness of middle-aged women, in this study, simple effect analysis is conducted respectively in relation to the aforementioned interaction variables which reach the significance level. Before the simple effect analysis, family health status and variables related to intergenerational housework support are classified in accordance with the above-mentioned classification method of parents' families. It can be seen from the results that when the health statuses of the middle-aged women, or those of fathers-in-law are different, the effect will be different. When the middle-aged women are in a healthy condition, those who help their parents-in-law's families do housework also have a higher happiness degree. However, in cases where the middle-aged women are in an unhealthy or normal condition, those who help their parents-in-law's families do housework tend to have a lower happiness degree; and in such cases, the middle-aged women who are in an unhealthy condition and 
Table 3. Regression analysis for middle-aged women perceived happiness (In-law's family).

\begin{tabular}{|c|c|c|c|c|c|}
\hline Variable & B & Beta & $\mathrm{t}$ & p-value & VIF \\
\hline \multicolumn{6}{|l|}{ Control variables } \\
\hline Age & 0.00 & 0.02 & 0.29 & 0.776 & 1.10 \\
\hline \multicolumn{6}{|l|}{ Work status $(0=$ Full-time $)$} \\
\hline Part-time & -0.09 & -0.04 & -0.56 & 0.575 & 1.14 \\
\hline Unemployed & 0.24 & 0.09 & 1.29 & 0.198 & 1.10 \\
\hline Gender role attitude & 0.03 & 0.03 & 0.39 & 0.701 & 1.10 \\
\hline SES (compared with other families in the society) & 0.09 & 0.08 & 1.11 & 0.269 & 1.07 \\
\hline \multicolumn{6}{|l|}{ Independent variable } \\
\hline Respondent's support to her parent in-law's housework & -0.16 & -0.09 & -1.24 & 0.218 & 1.34 \\
\hline Spouse's support to his parent's housework & 0.04 & 0.02 & 0.27 & 0.790 & 1.35 \\
\hline \multicolumn{6}{|l|}{ Moderator variable } \\
\hline Assessment of spouse's health status & 0.26 & 0.24 & $2.57^{\star}$ & 0.011 & 1.97 \\
\hline Assessment of self-health status & 0.25 & 0.24 & $2.72^{* *}$ & 0.007 & 1.71 \\
\hline Assessment of father in-law's health status & 0.03 & 0.04 & 0.34 & 0.736 & 2.59 \\
\hline Assessment of mother in-law's health status & 0.10 & 0.12 & 1.42 & 0.158 & 1.62 \\
\hline Respondent's support to her parent in-law's housework $\times$ Assessment of self-health status & 0.36 & 0.17 & $2.16^{*}$ & 0.032 & 1.38 \\
\hline Spouse's support to his parent's housework $\times$ Assessment of spouse's health status & 0.19 & 0.08 & 0.97 & 0.333 & 1.53 \\
\hline $\begin{array}{l}\text { Respondent's support to her parent in-law's housework } \times \text { Assessment of father in-law's } \\
\text { health status }\end{array}$ & -0.06 & -0.05 & -0.40 & 0.690 & 2.86 \\
\hline Spouse's support to his parent's housework $\times$ Assessment of father in-law's health status & -0.32 & -0.18 & $-2.04^{*}$ & 0.043 & 1.77 \\
\hline $\begin{array}{c}\text { Respondent's support to her parent in-law's housework } \times \text { Assessment of mother in-law's } \\
\text { health status }\end{array}$ & -0.01 & -0.00 & -0.04 & 0.972 & 2.06 \\
\hline Spouse's support to his parent's housework $\times$ Assessment of mother in-law's health status & -0.07 & -0.04 & -0.47 & 0.638 & 1.95 \\
\hline $\mathrm{R}^{2}$ & 0.29 & & & & \\
\hline Adjusted $\mathrm{R}^{2}$ & 0.22 & & & & \\
\hline F-vaule & $3.92^{* * *}$ & & & 0.000 & \\
\hline
\end{tabular}

${ }^{*} \mathrm{p}<0.05,{ }^{* *} \mathrm{p}<0.01,{ }^{* *} \mathrm{p} \leq 0.001$.

help their parents-in-law's families do housework achieve the lowest happiness degree (Figure 3). Besides, in cases where fathers-in-law are in an unhealthy condition and husbands provide housework support to the women's parents-inlaw's families, the middle-aged women have a higher happiness degree. If fathers-in-law are in a normal condition or healthy condition, the middle-aged women whose husbands help do housework in the women's parents-in-law's families achieve a lower happiness degree; in such case, if fathers-in-law are in a healthy condition, the middle-aged women whose husbands help do the housework of the women's parents-in-law's families achieve the lowest happiness degree (Figure 4). 




Respondent's support to her parent in-law's housework

Mean of Covariates: Age $=49.83$, Work status $($ Part-time $)=0.16$, Work status $($ Unemployed $)=0.11$, Gender role attitude $=2.81$, SES(compared with other families in the society) $=3.17$, Assessment of spouse' s health status $=3.70$, Assessment of mother in-law' s health status $=3.04$, Assessment of father in-law' s health status $=2.92$, Spouse' s support to his parent' $s$ house work $=0.28$

Figure 3. Interaction effect of respondent's support to her parent in-law's housework and assessment of self-health status.

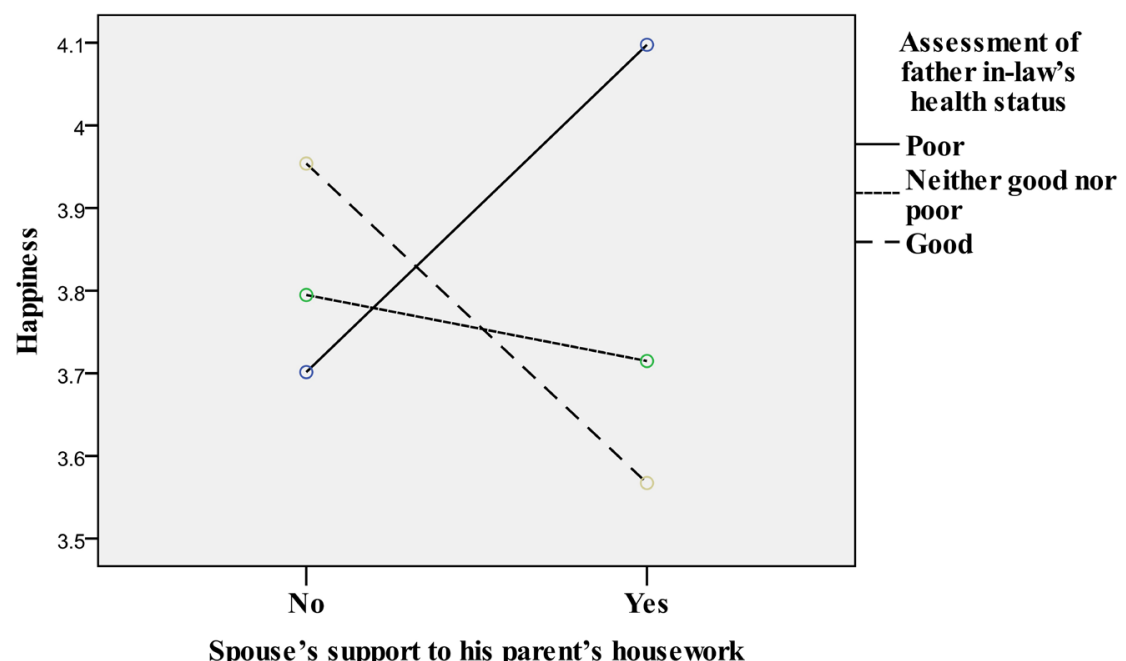

Mean of Covariates: Age $=49.83$, Work status $($ Part-time $)=0.16$, Work status (Unemployed) $=0.11$, Gender role attitude $=2.81$, SES (compared with other families in the society) $=3.17$, Assessment of spouse's health status $=\mathbf{3 . 7 0}$, Assessment of self-health status $=3.67$,

Assessment of mother in-law's health status $=\mathbf{3 . 0 4}$, Respondent's support to her parent inlaw's housework $=0.41$

Figure 4. Interaction effect of spouse's support to his parent's housework and assessment of father in-law's health status.

\section{Discussion}

\subsection{The Health Condition of Middle-Aged Women and Their Family Does Affect Their Happiness}

This study finds that when the health status of the middle-aged women, their husbands and their parents is better, their happiness degree is also relatively increased. It has been found in previous studies that when an individual is in an 
unhealthy state, s/he not only cannot have a normal daily life, but also needs the assistance of others in life, and thus s/he is restless and then will feel unhappy. In contrast, the improvement of health may enhance the happiness of individuals [36]. This study also finds that the respondents with better health status feel happier. It can be concluded that individuals' health is positively correlated with their psychological well-being.

Furthermore, the health status of spouses and mothers can also affect middle-aged women's happiness. This may be because women have traditionally been assigned the task of caring for the elderly and children in the family. Therefore, when spouses or the elderly in the family need to be cared for due to unhealthy status, middle-aged women are considered to be the right caregivers [29]. And if the female caregivers are not familiar with the use of the care resource mechanism or lack support in the care process, their psychological well-being is more likely to decline [37].

\subsection{The Moderating Effect of Family Health Status on the Relationship between Intergenerational Housework Support and Happiness of Middle-Aged Women Does Vary between Parents-in-Law's Families and Parents' Families}

\subsubsection{For Parents' Families}

This study finds that, compared with the middle-aged women whose mothers are in a healthy or normal condition, the happiness degree of those whose mothers' health is not good will decline as they help their parents' families do housework. This may be because such middle-aged women, who have their own family to look after, not only might worry about their mothers' health, but also need to bear most of the housework because of their mothers' poor health condition, and their pressure is reasonably higher; meanwhile, the middle-aged women are also under the shackles of the traditional norms of "daughters are water poured out of the family after they get married", which indicates that married women only need to do their best for the affairs of their husbands' family [38] and the housework of their parents' families should not belong to the business of the married daughters, and thus it is not difficult to understand why the happiness degree of the middle-aged women who complete housework for their parents' families becomes lower when their mothers, who are often regarded as the housekeepers of their parents' families, are in poor health. On the contrary, in cases where their mothers' health status is normal, middle-aged women may begin to realize that the health of their mothers is not as good as before. Middle-aged women helping to complete part of the housework in parents' families not only can provide some support for their mothers, but also do not have any impact on the housework in their own families' residences. Therefore, intergenerational housework support can increase their happiness degree. For the middle-aged women whose mothers are in good health, since such mothers are still fully capable of dealing with housework and the intergenerational housework assistance is not necessary; the middle-aged women's happiness may also decline 
when these middle-aged women help do the housework of their parents' families.

Furthermore, the middle-aged women's happiness degree will increase if they help their parents to complete the housework when their fathers have a poor health status. This may be due to the fact that middle-aged women's intergenerational housework support is providing their mothers with housework support so as to relieve their mothers' pressure, rather than acting as the people in charge of housework full-time, and thus the happiness degree is comparatively positive. Moreover, when the fathers are in a good or normal health condition, it also means that the fathers' life can be managed independently [39], and most of them do not need special care from others in housework, and relatively the middle-aged women are not required to provide intergenerational housework support. If women are required to provide housework support for their parents' families at this time, their happiness degree will decline.

\subsubsection{For Parents-in-Law's Families}

This study finds that the middle-aged women who are in good health and help their parents-in-law's families do housework achieve the highest happiness degree. The previous studies have found that after a Chinese daughter-in-law marries her husband, taking care of everything in parents-in-law's families, such as fulfilling filial duties to her parents-in-law for her husband, helping parents-inlaw's families do housework, caring for her parents-in-law's daily life, etc., shall be regarded as the indicators of virtue. Therefore, middle-aged women treat providing housework support to parents-in-law's families as a reasonable behavior related to respect for elders and filial piety [17]. As such, for healthy middle-aged women, helping their parents-in-law's families to do housework is in line with the role of a good daughter-in-law expected by the society, and their good health can have them provide full assistance, thus meaning they can feel happy. But for women with normal or poor health, their discomfort will increase the pressure of involvement in housework [40] and they also have to face the stereotyped expectations of the society regarding the role of a wife and a daughter-in-law, forcing them to do the housework of their parents-in-law's families; because of this, their happiness degree will naturally decline.

In addition, the middle-aged women whose fathers-in-law are in poor health and whose husbands help do the housework of their parents-in-law's families also have a higher happiness degree. As mentioned above, middle-aged women regard taking care of their parents-in-law's daily life as an internalized commitment, let alone when their fathers-in-law are in poor health. It is conceivable that women will be expected to undertake the housework of their parents-inlaw's families as a matter of course. In such cases, if the middle-aged women need to undertake the housework of their parents-in-law's families in addition to the housework of their own families, it can be imagined that they will suffer considerable pressure. Previous studies have also found that women's psychological well-being will decline when their husbands are less involved in housework as 
they have to undertake too much housework [41]. Therefore, if husbands can help complete the housework of the women's parents-in-law's families, it is more or less conducive to the improvement of the women's psychological well-being. On the other hand, when the fathers-in-law's health is good or normal, the assistance from husbands with the housework of parents-in-law's families will make the middle-aged women's happiness degree decrease. The reason for this may be that: most of the husbands provide assistance with the housework of women's parents-in-law's families mainly because they think that this is a manifestation of the filial piety of children in the culture context; however, most of the husbands still hold the view that the "husband is the breadwinner while wife is the homemaker" for their own family's housework, and they thus provide less assistance. Therefore, in cases where the fathers-in-law are healthy and do not need special care, but the husbands give intergenerational support, middle-aged women may feel less happy.

\section{Conclusions, Research Limitations and Suggestions}

In Chinese society, different types of intergenerational support and close network interaction are interwoven through marriage. Via marrying, women will be promoted to the best female roles, serving as the links to, and the caregivers of, their husbands' family network; moreover, sophisticating and contacting in such a case is one of the factors affecting the psychological well-being of married women [18]. However, with the change of society and the shift of time, does the female role in the cultural context still continue to bear the heavy burden of responsibility for the husbands' family in the current society in Taiwan? Or has the role played in the husbands' family been released with the infiltration of western thoughts? By investigating the moderating effect of family health on the relationship between intergenerational housework support and happiness of middle-aged women, it has been found that: neither middle-aged women's nor their spouses' assistance with intergenerational housework support to the parents' families or parents-in-law's families of the women has a direct impact on the happiness of middle-aged women. However, the health status of family members, especially the middle-aged women themselves, their spouses and their mothers, will have a direct impact on the happiness of the respondents and be positively correlated with their happiness. The moderating effect of family health status on the relationship between intergenerational housework support and happiness of middle-aged women does vary between parents-in-law's families and parents' families. The differences may be related to individuals' behaviors in culture trace [18], that is to say, women are entrusted with the responsibility of caring for their families and looking after their households by culture, but this does not mean that culture is the main factor causing women's unhappiness. On the contrary, in fact, the factors that affect women's psychological well-being may be that women themselves are not good at caring for their families, or that they find it difficult to bear the load due to lack of support or resources [29], and 
thus their psychological well-being declines. In particular, the care needs derived from the poor health of family members are different from the service and love for general housework [42], and it is more necessary to learn relevant care knowledge and skills to provide care of a better quality [43]. This study also finds that the health status of the family directly/indirectly affects the happiness of middle-aged women. Therefore, when issues such as the psychological well-being of middle-aged women are discussed in future, it is desirable to include variables related to family health status (such as physical and psychological health) in the discussion. In addition, when policies and programs to improve the life quality of middle-aged women are planned, it may also be necessary to consider not only self-health status but also other family health status. Since the female role in the cultural context still continues to bear the heavy burden of family care responsibility in Taiwan, the unhealthy families can cause women's stress even worse.

This study is a cross-sectional quantitative study, and thus it is impossible to conduct inference in accordance with a timing sequence in relation to the research results, while it is also not possible to develop a deep and detailed explanation in the form of a qualitative research project. However, different from previous studies, this study attempts to include intergenerational housework support and combine it with variables such as family health status, individual psychological well-being, etc. from the perspective of Chinese families' emphasis on intergenerational interaction, to explore the happiness and unhappiness in life of married middle-aged women. Although there is still a lot of room for discussion on the construction of the relevant knowledge in this study, it has opened up a new field for the discussion of middle-aged women's happiness.

\section{Acknowledgements}

We thank the Ministry of Science and Technology's program-“Intergenerational expectation and collaboration: an inquiry on a new mechanism of continued tradition across generations" for providing the data of this study.

\section{Conflicts of Interest}

The authors declare that there are no conflicts of interest.

\section{References}

[1] Sabatini, F. (2014) The Relationship between Happiness and Health: Evidence from Italy. Social Science \& Medicine, 114, 178-187. https://doi.org/10.1016/j.socscimed.2014.05.024

[2] Liu, B., Floud, S., Pirie, K., Green, J., Peto, R. and Beral, V. (2013) Does Happiness Itself Directly Affect Mortality? The Prospective UK Million Women Study. The Lance, 387, 874-881. https://doi.org/10.1016/S0140-6736(15)01087-9

[3] Donaldson, S.I., Dollwet, M. and Rao, M.A. (2015) Happiness, Excellence, and Optimal Human Functioning Revisited: Examining the Peer-Reviewed Literature Linked to Positive Psychology. The Journal of Positive Psychology, 10, 185-195. 
https://doi.org/10.1080/17439760.2014.943801

[4] Botha, F., Wouters, E. and Booysen, F. (2018) Happiness, Socioeconomic Status, and Family Functioning in South African Households: A Structural Equation Modelling Approach. Applied Research in Quality of Life, 13, 947-989.

https://doi.org/10.1007/s11482-017-9568-x

[5] Chiang, H.H. and Lee, T.S.H. (2018) Family Relations, Sense of Coherence, Happiness and Perceived Health in Retired Taiwanese: Analysis of a Conceptual Model. Geriatrics \& Gerontology International, 18, 154-160. https://doi.org/10.1111/ggi.13141

[6] Tseng, W.C. (2007) A Study of Folk Concepts of Good Life and Subjective Well-Being among College Students. Bulletin of Educational Psychology, 38, 417-441.

[7] Peng, T.C.P. and Huang, T.Y. (2012) Research on Happiness Index Building (Project No. G12111). Taiwan Foundation for Democracy, Taipei.

[8] Pinquart, M. and Sörensen, S. (2001) Gender Differences in Self-Concept and Psychological Well-Being in Old Age: A Meta-Analysis. Journal of Gerontology: Psychological Science, 56, 195-213. https://doi.org/10.1093/geronb/56.4.P195

[9] Hobbs, F.B. and Damon, B.L. (1996) 65 plus in the United States. U.S. Government Printing Office, Washington DC. https://doi.org/10.1037/e401512005-001

[10] Lee, C.F. and Tang, S.M. (2016) The Effects of Intergenerational Supports on the Happiness for the People Aged over 55 in Taiwan. Taiwan Journal of Gerontological Health Research, 12, 121-138.

[11] Thompson, L. and Walker, A.J. (1989) Gender in Families: Women and Men in Marriage, Work, and Parenthood. Journal of Marriage and the Family, 51, 845-871. https://doi.org/10.2307/353201

[12] Kobayashi, M., Okumura, T. and Usui, E. (2016) Sharing Housework between Husbands and Wives: How to Improve Marital Satisfaction for Working Wives in Japan. Journal of Labor Policy, 5, 18. https://doi.org/10.1186/s40173-016-0074-9

[13] Diener, E., Lucas, R.E. and Oishi, S. (2005) Subjective Well-Being: The Science of Happiness and Life Satisfaction. In: Snyder, C.R. and Lopez, S.J., Eds., Handbook of Positive Psychology, Oxford University Press, New York, 63-73.

[14] Yeh, K.H. (2009) Intergenerational Exchange Behaviors in Taiwan: The Filial Piety Perspective. Indigenous Psychological Research in Chinese Societies, 31, 97-141.

[15] Yeh, K.H. (2009) The Dual Filial Piety Model in Chinese Culture: Retrospect and Prospects. Indigenous Psychological Research in Chinese Societies, 32, 101-148.

[16] Bengtson, V.L. and Schrader, S.S. (1982) Parents-Child Relations. In: Mangen, D.J. and Peterson, W.A., Eds., Research Instruments in Social Gerontology, Vol. 2, University of Minnesota Press, Minneapolis, 115-128.

[17] Hsu, H.Y. (2013) The Cultural Expectations on the Role Daughters-in-Law Play in Family Care. Journal of Family Education and Counseling, 14, 33-52.

[18] Hsieh, Y.L. (2014) The Representation and Reality in Life Experience of Married Women. Journal of Contemporary Social Work, 6, 1-49.

[19] Wu, C.Y. and Chao, S.C. (2004) Utilizing Data from Multiple Family Members to Building Up Intergenerational Ambivalent Experiences. Formosa Journal of Mental Health, 17, 75-111.

[20] Lee, C.F. and Tang, S.M. (2013) The Father-in-Law and Son-in-Law Relationship: A Preliminary Study. Research in Applied Psychology, 27, 219-250.

[21] Chen, C.L. (2005) Parent-Child Living Arrangement under Intra- \& Inter-Household Members' Power Interaction. Journal of Housing Studies, 14, 51-82. 
[22] Huang, T.C., Li, J.R. and Chang, Y.M. (2010) Parentification and Self-Transformation in Intergenerational Relationships: A Study of Women in Early Adulthood. Indigenous Psychological Research in Chinese Societies, 33, 59-106.

[23] Huang, C.H. (2016) Family Mainstay: Baby Boomers' Intergenerational Support Type. Journal of Human Development and Family Studies, 17, 50-66.

[24] Kao, S.K. (2000) A Study of the Adjustment of Rural Elderly. Review of Agricultural Extension Science, 17, 27-57.

[25] Lee, C.F. and Tang, S.M. (2017) Relationship between Housework and Perceived Happiness of Middle-Aged and Older Women in Taiwan-The Moderating Effect of Health Condition. Health Care for Women International, 38, 1313-1326. https://doi.org/10.1080/07399332.2017.1354863

[26] Cloninger, C.R. and Zohar, A.H. (2011) Personality and the Perception of Health and Happiness. Journal of Affective Disorders, 128, 24-32. https://doi.org/10.1016/j.jad.2010.06.012

[27] Szinovacz, M.E. (2000) Changes in Housework after Retirement: A Panel Analysis. Journal of Marriage and the Family, 62, 78-92. https://www.jstor.org/stable/1566689 https://doi.org/10.1111/j.1741-3737.2000.00078.x

[28] Ko, C.F. (2002) Who Cares for the Elderly: A Comparative Study among EU Countries. Journal of Population Studies, 24, 1-32.

[29] Thomas, H., Britt, S. and Reidun, I. (2013) The Strains and Gains of Caregiving: An Examination of the Effects of Providing Personal Care to a Parent on a Range of Indicators of Psychological Well-Being. Social Indicators Research, 114, 323-343. https://doi.org/10.1007/s11205-012-0148-Z

[30] Rahtz, D.R., Sirgy, M.J. and Meadow, H.L. (2015) Exploring the Relationship between Healthcare Services Satisfaction and Life Satisfaction among the Elderly. In: Proceedings of the 1989 Academy of Marketing Science (AMS) Annual Conference, Springer International Publishing, Berlin, 531-536.

https://doi.org/10.1007/978-3-319-17055-8_108

[31] Lin, C.H., Chen, P.H. and Lin, H.S. (2010) A Panel Study on Factors Affecting the Changing Status of Depression among the Elderly in Taiwan. Journal of Population Studies, 41, 67-109.

[32] Shih, Y. (2017) The Impact of Using Social Welfare Services toward the Elders' Perception of Health Conditions and Life Satisfaction. Journal of Family Education Bimonthly, 65, 6-30.

[33] Matud, M.P., Bethencourt, J.M. and Ibáñez, I. (2014) Relevance of Gender Roles in Life Satisfaction in Adult People. Personality and Individual Differences, 70, 206-211. https://doi.org/10.1016/j.paid.2014.06.046

[34] Amato, P.R. and Booth, A. (1997) A Generation at Risk: Growing Up in an Era of Family Upheaval. Harvard University, Cambridge.

[35] Shiau, W.L. (2009) Best Practical Book of Multivariate Analysis-SPSS + LISREL. GOTOP Information Inc., Taipei.

[36] Chang, F.C. (2018) Education Can Enhance the Happiness? The Trust, Proud of Nation, Leisure, and Health of Mediating Factors Test. Journal of Social and Regional Development, 5, 75-105.

[37] Chu, T.Y., Lee, S.C., Wang, T.C., Hsieh, J.Y., Lee, C.Y. and Lin, H.L. (2010) A Qualitative Study of Burden among Female Caregivers. Taipei City Medical Journal, 7, 144-153.

[38] Yu, S.C. (2006) The Ending of the Traditional Women: A Study about "Nyu You 
Suo Gui" the Female's Education Pattern and the Reflection of Culture in Taiwanese Proverbs. Interdisciplinary Journal of Taiwan Library Administration, 2, 76-93. https://www.ntl.edu.tw/public/Attachment/99288454989.pdf

[39] Lee, P.G., Jackson, E.A. and Richardson, C.R. (2017) Exercise Prescriptions in Older Adults. American Family Physician, 95, 425-432.

[40] Schram, V.R. and Hafstrom, J.L. (1986) Family Resources Related to Wife's Time Inputs to Housework. Journal of Consumer Studies and Home Economic, 10, 235-245. https://doi.org/10.1111/j.1470-6431.1986.tb00122.x

[41] Frisco, M.L. and Williams, K. (2003) Perceived Housework Equity, Marital Happiness, and Divorce in Dual-Earner Households. Journal of Family Issues, 24, 51-73. https://doi.org/10.1177/0192513X02238520

[42] Tang, S.M. (1996) What Is Housework-A Study of the Nature of Housework. Journal of Living Science, 2, 209-236.

[43] Chiou, C.J., Chen, W.T. and Chen, I.P. (2005) The Support System for Family Caregivers of Chronically Ill Patients. The Journal of Long-Term Care, 9, 245-262. 